\title{
Conforto acústico: um panorama da exposição humana ao ruído ambiental em veículo do transporte público coletivo na capital de Mato Grosso
}

A preocupação com a exposição humana ao ruído ambiental de interiores é constante alvo de estudos, devido ao aumento de fontes geradoras de ruídos em meio urbano e o risco de efeitos psicofisiológicos a seus ocupantes. Neste cenário o ambiente veicular destinado ao transporte urbano de passageiros tem despertado grande interesse principalmente pelo fluxo constante de passageiros e por estarem sujeitos a eventos intrínsecos ao meio urbano como por exemplo o ruído de tráfego. Assim, este estudo avaliou a exposição humana ao ruído ambiental no interior de veículos do transporte público coletivo em Cuiabá, Mato Grosso, com o objetivo de investigar a salubridade e o conforto acústico. Foram monitoradas variações dos níveis de ruído $(\mathrm{dB}(\mathrm{A}))$ em três pontos predefinidos no interior veicular, nos horários matutino, vespertino e noturno em três dias úteis consecutivos no mês de agosto de 2018 e no mês de fevereiro de 2019. Para análise do conforto acústico utilizou-se o cálculo do Nível de ruído equivalente contínuo (Leq) e os Índices estatísticos de ruído L10 e L90, conforme Norma Técnica CETESB/L11.033 (1992), e os resultados comparados aos limites de tolerância estabelecidos pelo Ministério do Trabalho e Emprego (NR-15 e NR-17) e Organização Mundial de Saúde (OMS). Em todas as amostragens foram observados valores estimados de L10 e L90 acima de $90 \mathrm{~dB}(\mathrm{~A})$, divergindo aos limites preconizados pela OMS e NR-17, caracterizando o ambiente como muito ruidoso e acusticamente desconfortável. Isto posto, permite-se concluir que o ambiente é potencialmente prejudicial à saúde dos ocupantes do veículo, em particular ao posto de motorista.

\section{Acoustic comfort: an overview of human exposure to environmental noise in a public transport vehicle in the capital of Mato Grosso}

The concern with human exposure to environmental noise from interiors is a constant target of studies, due to the increase of noise-generating sources in urban areas and the risk of possible psychophysiological effects to its occupants. In this scenario, the vehicle environment for urban passenger transport has aroused great interest mainly for the constant flow of passengers and because they are subject to events intrinsic to the urban environment, such as traffic noise. Thus, this study evaluated human exposure to environmental noise inside public transport vehicles in Cuiabá, Mato Grosso, with the objective of investigating health iness and acoustic comfort. Variations in noise levels (dB(A)) were monitored in three predefined points in the vehicle interior, in the morning, evening and evening hours on three consecutive working days in August 2018 and February 2019. For acoustic comfort analysis, we used the calculation of the continuous equivalent noise level (Leq) and the statistical noise indexes $L 10$ and L90, according to CETESB/L11.033 (1992) Technical Standard, and the results compared to the tolerance limits established by the Ministry of Labor and Employment (NR-15 and NR-17) and the World Health Organization (WHO). In all samples, estimated values of L10 and L90 above $90 \mathrm{~dB}(\mathrm{~A})$ were observed, differing from the limits recommended by the WHO and NR-17, characterizing the environment as very noisy and acoustically uncomfortable. This is done, it is possible to conclude that the environment is potentially harmful to the health of the occupants of the vehicle, in particular the driver's seat.

Keywords: Buses; Noise; Continuous equivalent noise level.

Topic: Desenvolvimento, Sustentabilidade e Meio Ambiente

Reviewed anonymously in the process of blind peer.
Received: 02/07/2021

Approved: 28/07/2021
Roberta Daniela de Souza Lauxen da Silva (iD

Universidade de Cuiabá, Brasil

http://lattes.cnpq.br/6695057034861231

http://orcid.org/0000-0001-7268-2694

roberta.engenhariaambiental@gmail.com

Jonathan Willian Zangeski Novais (iD)

Universidade de Cuiabá, Brasil

http://lattes.cnpq.br/5665663207008673

http://orcid.org/0000-0002-6598-3138

jonathanzangeski@gmail.com

Marcelo Dias de Souza (D)

Universidade de Cuiabá, Brasil

http://lattes.cnpq.br/9474670068485343

http://orcid.org/0000-0002-4348-4645

marcelo.dias@florestal.eng.br

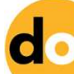

DOI: 10.6008/CBPC2179-6858.2021.007.0036

\author{
Pricila Juliana de Souza \\ Universidade de Cuiabá, Brasil \\ http://lattes.cnpq.br/1774304263302532 \\ pryjuliana@hotmail.com
}

Ana Carolina Freitas da Silva Taques (it)

Universidade Federal de Mato Grosso, Brasil

http://lattes.cnpq.br/4951797307571977

http://orcid.org/0000-0002-3903-3876

anacaroltaques@hotmail.com

Valdiclei Custódio Jorge

Universidade Federal de Mato Grosso, Brasi

http://lattes.cnpq.br/3535415890123524

valdiclei1@yahoo.com.br
Referencing this:

SILVA, R. D. S. L.; NOVAIS, J. W. Z.; SOUZA, M. D.; SOUZA, P. J.; TAQUES, A. C. F. S.; JORGE, V. C.. Conforto acústico: um panorama da exposição humana ao ruído ambiental em veículo do transporte público coletivo na capital de Mato Grosso. Revista Ibero Americana de Ciências Ambientais, v.12, n.7, p.405-413, 2021. DOI: http://doi.org/10.6008/CBPC2179-6858.2021.007.0036 


\section{INTRODUÇÃO}

A preocupação com a exposição humana ao ruído ambiental e a insalubridade acústica de interiores é constante tema de estudos, devido ao aumento de fontes geradoras de ruídos em meio urbano e o risco de danos psicofisiológicos a seus ocupantes (LEÃO et al., 2010; SILVA et al., 2012; SÁNCHEZ et al., 2019).

De acordo com a Organização Mundial de Saúde (WHO, 2004) essa sobreposição de sons que caracteriza o ruído ambiental urbano é considerada a terceira principal causa de poluição mundial. A OMS reitera ainda que o limiar de incomodidade situa-se entre a faixa de $50 \mathrm{~dB}(\mathrm{~A})$ e $55 \mathrm{~dB}(\mathrm{~A})$, já para a exposição a níveis superiores $70 \mathrm{~dB}(\mathrm{~A})$ além da perda auditiva induzida pelo ruído (PAIR) podem ocorrer outros sintomas como fadiga, cefaleias, tonturas e irritabilidade.

Em um cenário menos otimista, Pimentel-Souza (1992) alerta para os riscos de exposição contínua a níveis de ruídos a partir de $65 \mathrm{~dB}(\mathrm{~A})$, quando o organismo começa a sofrer estresse degenerativo com desequilíbrio bioquímico, aumentando os riscos de infarto do miocárdio e derrame cerebral.

Dentre os ambientes internos a serem avaliados, o veicular utilizado para transporte urbano de passageiros, se destaca principalmente pela elevada movimentação de pessoas, por estarem em curso constante e sujeitos a eventos intrínsecos ao meio urbano como por exemplo o ruído de tráfego, e que segundo Bistafa (2011), constitui uma das principais fontes de poluição ambiental.

Ressalta-se que as características construtivas destes veículos, como por exemplo a localização do motor na parte dianteira, contribuem de forma direta para as condições de conforto acústico. Silva et al. (2005) em seus estudos no ambiente veicular do transporte coletivo, constataram que o nível de pressão sonora semanal para o posto de motorista foi maior quando o motor se localizava na parte dianteira.

Baseado nestes aspectos é igualmente necessário considerar os efeitos do risco à exposição dos ocupantes a diferentes níveis de poluição sonora no interior veicular, pois de acordo com a pesquisa realizada por Chan et al. (2003), os habitantes de regiões urbanas passam aproximadamente 7\% do seu tempo diário em meios de transporte.

A identificação de fontes de ruídos e a adoção de medidas corretivas para sua atenuação nestes ambientes pode contribuir para diminuição do estresse e corroborar com o aumento da atenção e a melhoria do desempenho do condutor além da segurança do motorista e passageiro (FARZANEH et al., 2008).

Uma das ferramentas disponíveis para avaliação do ruído ambiental são os descritores acústicos, que se baseiam em limites máximos normatizados, podendo variar de acordo com a legislação de cada país. Dentre os descritores de ruído mais utilizados pode-se citar o nível de ruído equivalente contínuo ( $L_{\text {eq }}$ e os Índices estatísticos de ruído $L_{10}$ e $L_{90}$, pois possibilitam observar as variações de amplitudes sonoras e auxiliar na interpretação dos valores obtidos, corroborando para a avaliação do grau de incomodidade, além de possíveis danos psicofisiológicos que ocupantes de um dado ambiente estão expostos durante o tempo de permanência.

Isto posto, este estudo objetivou avaliar a exposição humana ao ruído ambiental no interior de veículos do transporte público coletivo em Cuiabá, Mato Grosso, visando investigar a salubridade e o 
conforto acústico.

\section{MATERIAIS E MÉTODOS}

O estudo foi realizado no munícipio de Cuiabá, capital do Estado de Mato Grosso, considerada como um dos principais polos de desenvolvimento da região Centro-Oeste do Brasil, na qual está situado na porção centro-sul do estado sob as coordenadas $15^{\circ} 35^{\prime} 46^{\prime \prime}$ de latitude Sul, e $56^{\circ} 5^{\prime} 48^{\prime \prime}$ de longitude Oeste.

De acordo com o último levantamento censitário Cuiabá possui uma população de aproximadamente 551.098 habitantes (IBGE, 2010), deste total, a Associação Mato-grossense dos Transportadores Urbanos estima que 200 mil pessoas fazem uso diariamente da frota do transporte coletivo que é formada atualmente por 369 ônibus, distribuída em mais 73 linhas realizando itinerários diários, bairros-centro-destino e viceversa (SOUZA, 2018).

Para realização da pesquisa optou-se por uma linha que percorresse bairros periféricos da cidade com destino a região central (figura 1). 0 itinerário da respectiva linha de ônibus possui aproximadamente $18 \mathrm{~km}$ e leva em média 60 minutos para realização do percurso completo (centro-bairro-centro). Neste contexto, encontra-se inserido o objeto de estudo que compreende ao ambiente interno do ônibus, sua caracterização estrutural e planta baixa simplificada estão representadas pela Figura 2.
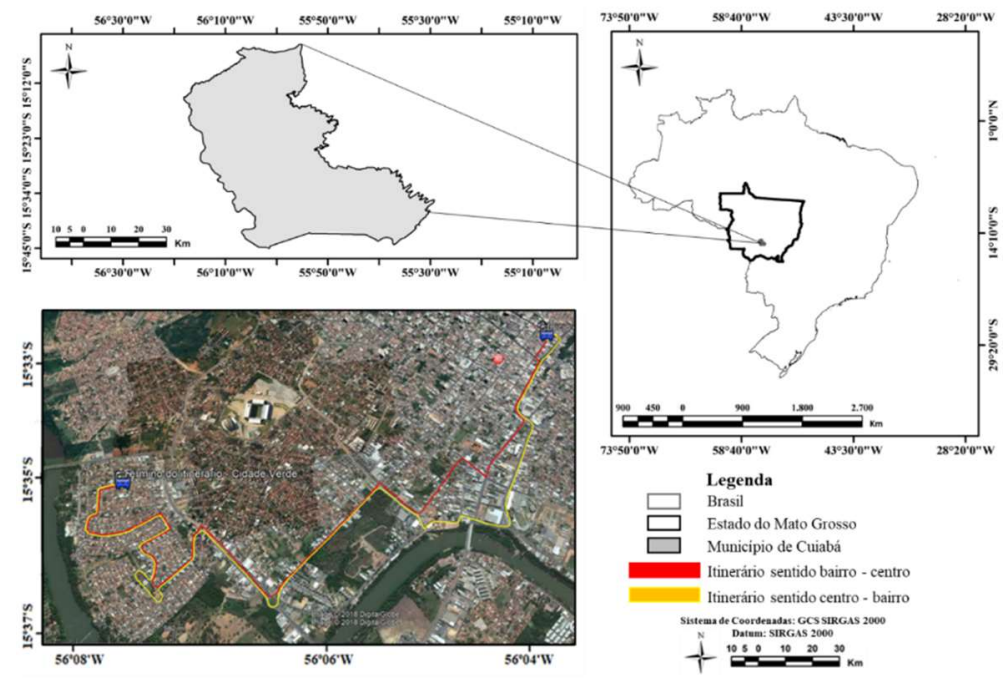

Figura 1: Localização Geográfica do município de Cuiabá, MT, Brasil. Com destaque para o Itinerário da linha de ônibus entre bairros (Centro - terminal Cidade Verde - Centro), o traçado amarelo refere-se a rota sentido centro-bairro e o vermelho sentido bairro-centro. Fonte: Adaptado de Google Earth (2019).

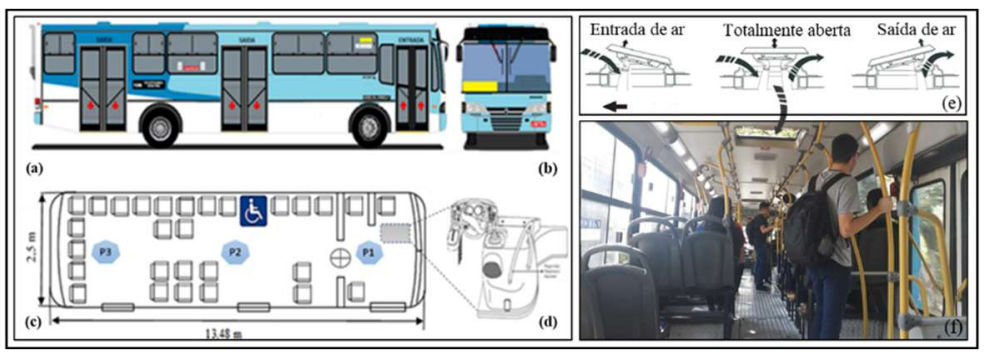

Figura 2: Caracterização estrutural do ônibus: (a) visão lateral do veículo; (b) visão frontal do veículo; (c) planta baixa simplificada com dimensões do veículo e especificações técnicas e (d) posicionamento do motor na parte frontal do veículo, (e) escotilhas e posicionamento correto para efetiva renovação e/ou ventilação de ar no salão de passageiros e (f) entradas de ar no teto do ônibus em estudo. Fonte: Adaptado de MARCOPOLO (2014). 
Como característica estrutural, o veículo possui três portas (duplas) pivotadas elétricas conforme Figura 2 (a), estando a segunda (disposta na parte central do veículo) equipada com sistema de rampa elevatória para os cadeirantes. Ademais, o motor está posicionado na parte dianteira (d) próximo à área do motorista, além de capacidade para transporte de 32 passageiros sentados e 44 passageiros em pé.

Cabe ressaltar que como os veículos da respectiva linha possuem as mesmas características construtivas (marca/modelo), a amostragem foi realizada ao acaso, ou seja, não se absteve a um único veículo, pois a intenção do estudo é recolher informações dos níveis de ruído em que os ocupantes destes ambientes encontram-se expostos.

Foram predefinidos três pontos no interior do ônibus (Figura 2 c) para monitoramento dos níveis de ruído $(\mathrm{dB}(\mathrm{A}))$, estando o primeiro $(\mathrm{P} 1)$ localizado na área do motorista, e os demais distribuídos após a catraca (P2 e P3), impreterivelmente posterior as portas de acesso (entrada-saída) não obstruindo o corredor e possibilitando a circulação no salão de passageiros durante o itinerário.

No intuito de otimizar resultados e obter maior controle sobre o erro experimental durante o procedimento de amostragem, utilizou-se a técnica de controle que consiste em subdividir o tratamento em blocos homogêneos para que o dimensionamento da amostra transcorra de maneira a aleatorizar os pontos de coleta no interior do veículo em função dos horários de amostragem, realizadas em três dias úteis consecutivos durante períodos distintos do ano, no mês de agosto de 2018 e no mês de fevereiro de 2019, em três turnos, matutino (início das coletas às $8 \mathrm{~h}$ ), vespertino (às $14 \mathrm{~h}$ ) e noturno (às $18 \mathrm{~h}$ ), com registros a cada segundo durante o tempo de realização do itinerário completo (centro-bairro-centro) de aproximadamente 60 minutos.

Contou-se com um Medidor de Nível de Pressão Sonora (NPS, Decibelímetro) Modelo DT-8852 com Datalogger integrado fabricado pela empresa CENTRALBRASIL (recomendado pela Norma IEC61672-1 CLASSE 2), realizando medições na faixa entre $30 \mathrm{~dB}$ e $130 \mathrm{~dB}$, ajustado em faixa de ponderação " $\mathrm{A}$ " (que mais se aproxima da audibilidade humana) para nível de ruído normal (precisão \pm 1.4 ), programado para respostas lentas modo Slow.

Foi estabelecida a altura média de $120 \mathrm{~cm}$ em relação ao piso para manuseio do equipamento pelo pesquisador, levando em consideração as medidas antropométricas de um indivíduo sentado definidas pelo Instituto Nacional de Tecnologia (FERREIRA, 1988). Após concluídas as coletas no interior veicular, iniciou-se o tratamento e a análise dos dados com a aplicação do cálculo do nível de ruído equivalente contínuo ( $L_{\text {eq }}$ e os Índices estatísticos de ruído $L_{10}$ e $L_{90}$, baseada nos critérios fixado pela Norma Técnica CETESB/L11.033 (São Paulo, 1990).

Segundo Murgel (2007) o L10, é o índice mais utilizado para estudo de ruído ambiental, também considerado como o ruído de pico e está presente em $10 \%$ do tempo de medição. Já o L90, refere-se ao nível de ruído de fundo pois representa o valor acima do qual os demais níveis permanecem $90 \%$ do tempo de medição (GERGES, 2000).

Após cumprir os pré-requisitos citados na Norma CETESB/L11.033 para a identificação dos Índices estatísticos $L_{10}$ e $L_{90}$, foi calculado o Nível de ruído equivalente contínuo ( $L_{\text {eq }}$ ) do ambiente, conforme a 
Equação (1):

$$
\boldsymbol{L e q}=0,010 *\left(L_{10}-L_{90}\right)^{2}+0,50\left(L_{10}+L_{90}\right)
$$

Em que:

Leq - Nível de ruído equivalente contínuo dB(A); $\mathrm{L}_{10}$ - Índices estatísticos a $10 \%$ do tempo de medição considerado; L9o - Índices estatísticos a 90\% do tempo de medição considerado.

Os resultados foram comparados aos valores limite normatizados pela NR-15 (85 dB(A)), NR-17 (para conforto acústico de até $65 \mathrm{~dB}(\mathrm{~A})$ ) e OMS (até $55 \mathrm{~dB}(\mathrm{~A})$ ) para verificação da adequabilidade acústica do ônibus.

Para a análise estatística, os dados amostrados foram submetidos à análise de variância (ANOVA) para de verificar a ocorrência de diferença significativa entre as médias dos fatores estudados (pontos de amostragem $\mathrm{x}$ horários de coletas), sendo estes comparados a um nível de significância de $5 \%$ aplicando o Teste de Tukey. Para tanto, utilizou-se o software R, que de acordo com R Development Core Team (2019) trata-se de um software livre sob os termos da General Public. License da Free Software Foundation - GNU, em forma de código fonte para desenvolvimento de cálculos estatísticos.

\section{RESULTADOS E DISCUSSÃO}

Os resultados dos valores obtidos de ruído, por medição e estimados durante os dias analisados, nos pontos preditos em função dos horários do dia podem ser visualizados na tabela 1.

Tabela 1: Níveis de ruídos registrados no interior veicular em função dos horários, nos meses de agosto 2018 e fevereiro 2019 e valores estimados dos índices estatísticos $L_{10}$, $L_{90}$ e $L_{e q}$ e médias do período.

\begin{tabular}{|c|c|c|c|c|c|c|c|c|}
\hline \multicolumn{9}{|c|}{ Nível de Ruído (dB(A)) } \\
\hline \multicolumn{9}{|c|}{ Período do Ano } \\
\hline \multirow[t]{2}{*}{ Dia/Horário/Ponto } & \multicolumn{4}{|l|}{ Agosto } & \multicolumn{4}{|l|}{ Fevereiro } \\
\hline & $\begin{array}{l}\text { Mín. } \\
\text { Máx. }\end{array}$ & $L_{10}$ & $L_{90}$ & Leq & $\begin{array}{l}\text { Mín. } \\
\text { Máx. }\end{array}$ & $L_{10}$ & $L_{90}$ & Leq \\
\hline M P1 & $68,4-95,1$ & 87,8 & 76,4 & 83,4 & $72,1-96,3$ & 82,9 & 75,8 & 79,9 \\
\hline $1^{\circ}$ Dia V P2 & $59,1-91,0$ & 82,3 & 71,4 & 88,7 & $\begin{array}{l}67,1- \\
107,3 \\
\end{array}$ & 85,9 & 72,6 & 81,0 \\
\hline N P3 & $68,6-102,3$ & 83,4 & 71,1 & 79,0 & $60,8-93,5$ & 80,8 & 66,9 & 75,8 \\
\hline M P2 & $72,1-95,1$ & 82,9 & 75,8 & 79,9 & $68,5-94,7$ & 84,5 & 73,0 & 80,1 \\
\hline $2^{\circ}$ Dia V P3 & $57,9-92,1$ & 81,7 & 69,5 & 77,1 & $63,2-89,2$ & 80,7 & 69,8 & 76,4 \\
\hline N P1 & $60,9-94,1$ & 83,1 & 72,3 & 78,9 & $74,9-95,6$ & 86,5 & 77,8 & 82,9 \\
\hline M P3 & $65,5-91,9$ & 81,4 & 68,0 & 76,5 & $72,2-92,1$ & 84,6 & 74,5 & 80,5 \\
\hline $3^{\circ}$ Dia V P1 & $71,1-95,2$ & 84,7 & 76,7 & 81,3 & $67,5-95,0$ & 83,2 & 70,7 & 78,1 \\
\hline N P2 & $63,6-94,8$ & 83,5 & 69,7 & 78,5 & $64,4-93,5$ & 83,3 & 69,0 & 78,2 \\
\hline Média Período & - & 83,4 & 72,3 & 80,3 & - & 83,6 & 72,2 & 79,2 \\
\hline
\end{tabular}

Legenda: Min. = Mínimo; Máx.= Máximo; $\mathrm{M}=$ Matutino; $\mathrm{V}=$ Vespertino; $\mathrm{N}=$ Noturno; $\mathrm{P}=$ Pontos experimentais; (localização dos pontos: P1 = área do motorista; P2 = centro do salão de passageiros; P3 = final do salão de passageiros); L10 = Índices estatísticos a $10 \%$ do tempo de medição considerado; $\mathbf{L} 90$ = Índices estatísticos a $90 \%$ do tempo de medição considerado; Leq = Nível de ruído equivalente contínuo $\mathrm{dB}(\mathrm{A})$.

Em função da tabela 1, observa-se que dentre os valores de $L_{\text {eq }}$ estimados nas amostragens realizadas no mês de agosto, o nível de ruído ultrapassou o limite preconizado pela NR-15 (85 dB(A)) no P2 do primeiro dia $(88,7 \mathrm{~dB}(\mathrm{~A}))$, em que a média aritmética estimada de $L_{\text {eq }}$ foi $80,3 \mathrm{~dB}(A)$, estando em consonância ao limite preconizado pela NR-15 (85 dB(A)). Porém, em relação a incomodidade, que se baseia nos valores dos índices estatísticos $L_{10}$ (ruído de pico) e $L_{90}$ (ruído de fundo) durante o tempo de amostragem, nota-se que ambas as 
médias (83,4 $\mathrm{dB}(\mathrm{A})$ e $72,3 \mathrm{~dB}(\mathrm{~A})$, respectivamente) excederam os níveis aceitáveis recomendados pela NR17 (até $65 \mathrm{~dB}(\mathrm{~A})$ ) e OMS (até $55 \mathrm{~dB}(\mathrm{~A})$ ).

Para as amostragens realizadas no mês de fevereiro, a média aritmética do Leq no período chuvoso foi 79,2 dB(A), obedecendo o limite de tolerância da NR-15 (85 dB(A)). Cabe ressaltar que não houve registros de $L_{\text {eq }}$ superiores aos de referência. Por outro lado, os valores médios dos índices estatísticos $L_{10}$ (ruído de pico, 83,6 dB(A)) e $\mathrm{L}_{90}$ (ruído de fundo, 72,2 dB(A)) excederam os níveis aceitáveis pela NR-17 e OMS.

Pesquisadores como Guedes et al. (1997) afirmam ainda que, além dos limites normativos, um ambiente também pode ser classificado de acordo com o nível de ruído estimado, sendo "pouco ruidosos" quando o $\mathrm{L}_{\text {eq }}$ encontrado é menor ou igual a $65 \mathrm{~dB}(\mathrm{~A})$, "ruidosos" entre $65 \mathrm{~dB}(\mathrm{~A})$ e $75 \mathrm{~dB}(\mathrm{~A})$ e "muito ruidosos" quando ultrapassa $75 \mathrm{~dB}(\mathrm{~A})$. De posse dessas informações (Tabela 1), verificou-se que em todos os pontos avaliados os resultados do Leq durante as amostragens, em ambos os meses, o limite de $75 \mathrm{~dB}(\mathrm{~A})$ foi excedido, classificando o ambiente como "muito ruidoso".

Em relação a incomodidade, com base nos valores dos índices estatísticos $L_{10}$ (ruído de pico) e $L_{90}$ (ruído de fundo), todos os níveis sonoros estimados divergiram aos valores de referência recomendados pela NR-17 (até $65 \mathrm{~dB}(\mathrm{~A})$ ) e OMS (até $55 \mathrm{~dB}(\mathrm{~A})$ ) para incômodo sério, logo, além de inadequados os níveis sonoros expõem os ocupantes do ônibus a situações desconfortáveis, podendo contribuir para o desenvolvimento de distúrbios psicofisiológicos.

Para a análise estatística, foram utilizadas letras para comparação dos valores estimados, sendo maiúsculas nas linhas e minúsculas nas colunas. Diante dos resultados da análise estatística aplicada às variações de ruído, observa-se a ocorrência de diferenças significativas $(p<0,05)$ entre os fatores pontos nos horários analisados em ambos os períodos do ano (Tabela 2).

Tabela 2: Análise Fatorial e valores médios de Ruído dos pontos experimentais no interior veicular em função dos horários, nos períodos seco e chuvoso.

\begin{tabular}{|c|c|c|c|c|c|c|}
\hline \multicolumn{7}{|c|}{ Análise Fatorial de Ruído (dB(A)) } \\
\hline \multicolumn{7}{|c|}{ Período do Ano } \\
\hline \multirow[t]{2}{*}{ Horário } & \multicolumn{3}{|l|}{ Agosto } & \multicolumn{3}{|l|}{ Fevereiro } \\
\hline & $P 1$ & $P 2$ & P3 & $P 1$ & $P 2$ & P3 \\
\hline Matutino & $77,18 \mathbf{B b}$ & $79,05 \mathrm{Aa}$ & $74,57 \mathrm{Cb}$ & $79,92 \mathbf{A b}$ & $79,61 \mathrm{Aa}$ & $79,54 \mathrm{Aa}$ \\
\hline Vespertino & $79,96 \mathbf{A a}$ & $77,11 \mathbf{B b}$ & $76,28 \mathrm{Ba}$ & $76,85 \mathrm{Bc}$ & $79,57 \mathrm{Aa}$ & $75,75 \mathrm{Bb}$ \\
\hline Noturno & 78,44 Aab & $77,05 \mathrm{Ab}$ & $77,43 \mathrm{Aa}$ & $82,00 \mathrm{Aa}$ & $76,71 \mathbf{B b}$ & $74,85 \mathbf{C b}$ \\
\hline CV (\%) & \multicolumn{3}{|l|}{3,24} & \multicolumn{3}{|l|}{3,37} \\
\hline p-value & \multicolumn{3}{|l|}{$<0,01$} & \multicolumn{3}{|l|}{$<0,01$} \\
\hline
\end{tabular}

Legenda: Médias seguidas de mesma letra Maiúscula nas linhas e minúscula nas colunas não diferem estatisticamente entre si ao nível de $5 \%$ de probabilidade pelo teste de Tukey.

Nos resultados das amostragens do mês de agosto (Tabela 2) no horário matutino, ocorreram diferenças estatísticas entre todos os pontos, porém, o P2 sobressaiu-se aos demais com o maior valor médio significativo. No horário vespertino o P1 diferiu-se estatisticamente aos demais, devido aos maiores valores médios significativos do horário amostrado. No horário noturno não ocorreram diferenças estatísticas entre os pontos nos horários analisados.

No mês de fevereiro, não foram observadas diferenças significativas entre os valores médios dos pontos no horário matutino. Em relação ao horário vespertino o P2 diferiu-se dos demais pontos com maior 
valor médio significativo. Já no horário noturno apesar de todos os pontos diferirem-se estatisticamente, o P1 sobressaiu-se aos demais com o maior valor médio significativo.

Ressalta-se que em ambos os meses analisados os pontos P1 e P2 destacaram-se com os maiores valores médios significativos dentre os horários analisados, e pode estar relacionada a combinação de ruídos e vibrações de fontes emissoras no interior do veículo tais como motor (P1) localizado na parte dianteira do veículo (área do motorista) e até mesmo dos passageiros que permanecem no reduzido espaço que antecede a catraca para aquisição das passagens em pontos específicos do itinerário, do sistema de rampa elevatória para os cadeirantes (P2) instalado nas portas de embarque/desembarque centrais e vibração das janelas.

Quanto ao ruído originário do motor, estudos realizados por Silva et al. (2005) constataram que o valor médio dos níveis equivalentes de ruído dos veículos com motor dianteiro é quase cinco vezes maior que o valor médio do ônibus com o motor na traseira.

Outro estudo realizado por Fernandes (2005) no município de Bauru localizado no estado de São Paulo, avaliou o nível de ruído e o risco de perda auditiva dos motoristas e cobradores de ônibus urbano, em que o nível médio encontrado foi $90 \mathrm{~dB}(\mathrm{~A})$ para motoristas e $87 \mathrm{~dB}(\mathrm{~A})$ para cobradores, resultando na caracterização de insalubridade ocupacional aos colaboradores e no risco de perda auditiva induzida pela exposição contínua ao ruído.

Em se tratando do ruído proveniente das janelas, pode ter como causa a vibração dos vidros no interior das calhas pode estar relacionado ao desgaste natural que inclui o ressecamento das borrachas de vedação (também chamadas de guarnições) em seu envoltório. No quesito ruído interno de veículos fabricados especificamente para transporte urbano de passageiros, a NBR 15570 enfatiza que os níveis não devem ultrapassar $85 \mathrm{~dB}(\mathrm{~A})$ em qualquer regime de rotação do motor (ABNT, 2009). No entanto, em todas as amostragens foram identificados valores máximos superiores ao normatizado.

Oliveira et al. (2007) enfatizam que a exposição contínua ao ruído pode ter consequências como irritação e dificuldades de concentração, dores de cabeça, aumento da pressão arterial, problemas digestivos e em virtude do estresse pode inclusive contribuir ao desenvolvimento de problemas cardiovasculares, além de perda auditiva.

\section{CONCLUSÕES}

Em todas as amostragens foram registrados valores acima dos $90 \mathrm{~dB}(\mathrm{~A})$ caracterizando o ambiente como muito ruidoso, acusticamente desconfortável e potencialmente prejudicial à saúde dos ocupantes do veículo, em particular ao posto de motorista.

Apesar de situações de desconforto acústico terem sido constatadas em todos os pontos amostrados, a área do motorista demanda maior preocupação devido ao tempo de permanência no local para execução de sua atividade laboral, estando mais propenso ao desenvolvimento de patologias oriundas a exposição a diferentes tipos e intensidades de ruídos que podem levar a diminuição do déficit de atenção e ao aumento do risco de acidente, consequentemente, comprometendo a segurança de todos os ocupantes do veículo. 
AGRADECIMENTOS: O presente trabalho foi realizado com apoio da Coordenação de Aperfeiçoamento de

Pessoal de Nível Superior - Brasil (CAPES) - Código de Financiamento 001.

\section{REFERÊNCIAS}

ABNT. Associação Brasileira de Normas Técnicas. ABNT-NBR 15570: Transporte - Especificações técnicas para fabricação de veículos de características urbanas para transporte coletivo de passageiros. Rio de Janeiro: ABNT, 2009.

BISTAFA, S. R.. Acústica Aplicada ao Controle do Ruído. 2 ed. São Paulo: Blucher, 2011.

BRASIL. Ministério do Trabalho e Emprego. Norma Regulamentadora N. 15. Dispõe sobre atividades e operações insalubres. Ministério do Trabalho e Emprego. Brasília: MTE, 1978.

BRASIL. Ministério do Trabalho e Emprego. Norma Regulamentadora N. 17. Dispõe sobre Ergonomia. Ministério do Trabalho e Emprego. Brasília: MTE, 1978.

CHAN, A. T.; CHUNG, M. W.. Indoor-outdoor air quality relationships in vehicle: effect of driving environment and ventilation modes. Atmospheric Environment, v.37, n.27, p.3795-3808, 2003.

FARZANEH, Y.; TOOTOONCHI, A. A.. Controlling automobile thermal comfort using optimized fuzzy controller. Applied Thermal Engineering, v.28, p.1906-1917, 2008.

FERNANDES, J. C.. Acústica e ruídos. Apostila da Disciplina Higiene do Trabalho, modulo: Acústica e Ruídos. Curso de Especialização em Engenharia de Segurança do Trabalho. Bauru: UNESP, 2005.

FERREIRA, D. M. P.. Pesquisa Antropométrica e Biomecânica dos Operários da Indústria de Transformação. 1988.

IBGE. Instituto Brasileiro de Geografia e Estatística. Censo Demográfico. IBGE, 2010.

GERGES, S. N. Y.. Ruído: fundamentos e controle. 2 ed. Florianópolis: NR, 2000.

GUEDES, M.; VALADAS, B.; COELHO, J. B.. Exposição ao ruído da população em Portugal. Amadora: Instituto do Ambiente, 1997.

LEÃO, R. N.; DIAS, F. A. M.. Perfil audiométrico de indivíduos expostos ao ruído atendidos no núcleo de saúde ocupacional de um hospital do município de Montes Claros, Minas Gerais. Revista CEFAC, v.12, n.2, p.242-249, 2010.
MARCOPOLO. Departamento de Assistência Técnica Marcopolo S.A. Manual de Operação e Manutenção. MARCOPOLO, 2014.

MURGEL, E.. Fundamentos de acústica ambiental. Senac, 2007.

OLIVEIRA, A. C. F.; PINHEIRO, J. Q.. Indicadores psicossociais relacionados a acidentes de trânsito envolvendo motoristas de ônibus. Psicologia em Estudo, v.12, n.1, p.171-178, 2007.

PIMENTEL-SOUZA, F.. Efeitos da poluição sonora no sono e na saúde em geral: ênfase urbana. Revista Brasileira de Acústica e Vibrações, v.10, p.12-22, 1992.

R DEVELOPMENT CORE TEAM. R: A language and environment for statistical computing. Vienna: R Foundation for Statistical Computing, 2019.

SÁNCHEZ, O. J. T.; ECHAVARRÍA, G. B.; LIZÁRRAGA, S. L. O.; FRAIRE, R. O. R.; RAMÍREZ, K. S.. Ruido en el ambiente laboral y efectos en sistema cardiovascular en operadores de transporte público. Salud de los Trabajadores, v.27, n.2, p.175-181, 2019.

SÃO PAULO. Companhia de Saneamento do Estado de São Paulo. CETESB L11033: Instrução Técnica para aplicação da Norma Brasileira NBR 10.151. São Paulo: CETESB, 1990.

SILVA, L. F.; MENDES, R.. Exposição combinada entre ruído e vibração e seus efeitos sobre a audição de trabalhadores. Revista de Saúde Pública, v.39, p.9-17, 2005.

SILVA, L. F.; CORREIA, F. N.. Avaliação da exposição de passageiros ao ruído no interior de ônibus do transporte público do município de Itajubá. Revista CEFAC, v.14, n.1, p.57-64, 2012. DOI: http://doi.org/10.1590/S151618462011005000115

SOUZA, L.. Ações de fiscalização do transporte público são intensificadas para melhor atender os usuários. Associação Mato-grossense dos Transportadores Urbanos - MTU. Cuiabá: AMTU, 2018.

WHO. World Health Organization. Occupational noise: Assessing the burden of disease from work-related hearing impairment at national and local levels. Geneva:

Environmental Burden of Disease Series, 2004.

A CBPC - Companhia Brasileira de Produção Científica (CNPJ: 11.221.422/0001-03) detém os direitos materiais desta publicação. Os direitos referem-se à publicação do trabalho em qualquer parte do mundo, incluindo os direitos às renovações, expansões e disseminaç̃oes da contribuição, bem como outros direitos subsidiários. Todos os trabalhos publicados eletronicamente poderão posteriormente ser publicados em coletâneas impressas sob coordenação da Sustenere Publishing, da Companhia Brasileira de Produção Científica e seus parceiros autorizados. Os (as) autores (as) preservam os direitos autorais, mas não têm permissão para a publicação da contribuição em outro meio, impresso ou digital, em português ou em tradução. 